\title{
General method for the fabrication of hollow microcapsules with adjustable shell compositions
}

Nan Ren, Bo Wang, You-hao Yang, Ya-hong Zhang, Wu-li Yang, Ying-hong Yue, Zi Gao and Yi Tang

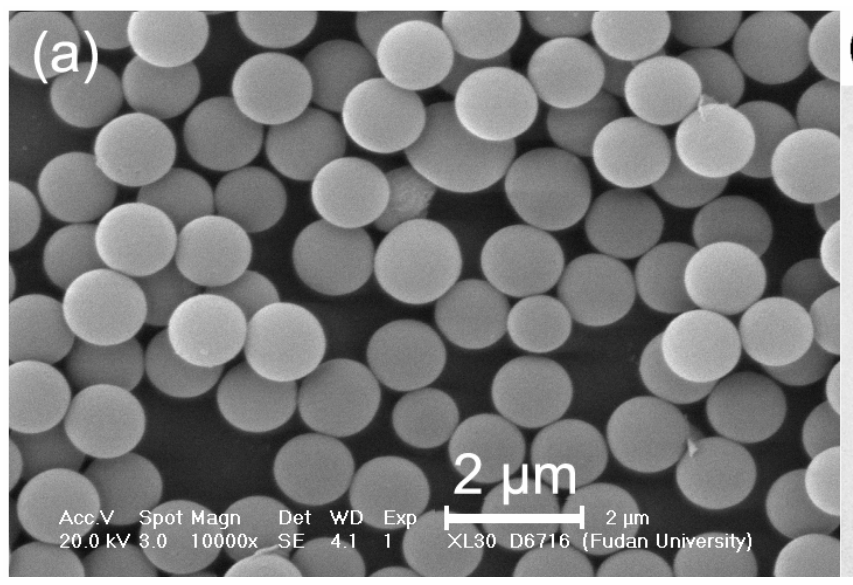

(b)

$0.2 \mu \mathrm{m}$

Figure S1. SEM (a) and TEM (b) images of MSSs.

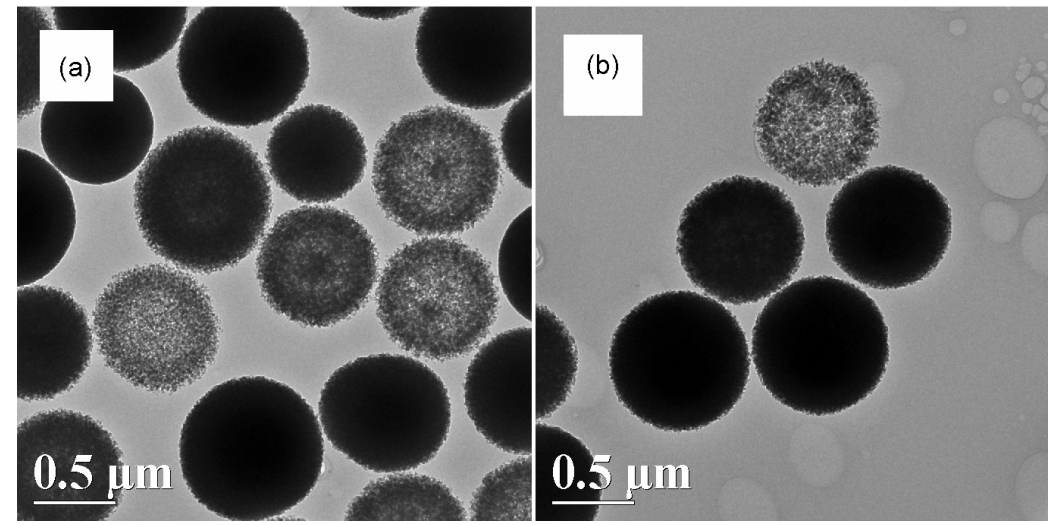

Figure S2. TEM images of the samples obtained by treating PSS (a) and PVP (b) coated MSSs with $1 \mathrm{M}$ ammonia solution. 


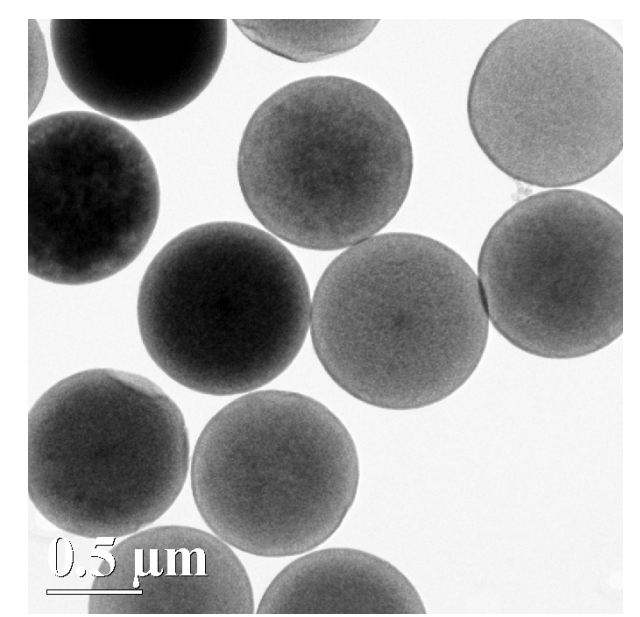

Figure S3. TEM image of the sample obtained by treating zirconia pre-incorporated MSSs with $3 \mathrm{M}$ ammonia solution at $100{ }^{\circ} \mathrm{C}$. 